\title{
Connectivity Analysis of Millimeter-Wave Device-to-Device Networks with Blockage
}

\author{
Haejoon Jung ${ }^{1}$ and In-Ho Lee ${ }^{2}$ \\ ${ }^{1}$ The Department of Information and Telecommunication Engineering, Incheon National University, Incheon 22012, Republic of Korea \\ ${ }^{2}$ The Department of Electrical, Electronic and Control Engineering, Hankyong National University, Anseong 456-749, Republic of Korea \\ Correspondence should be addressed to In-Ho Lee; ihlee@hknu.ac.kr
}

Received 3 August 2016; Revised 8 October 2016; Accepted 17 October 2016

Academic Editor: Mohammad Abdul Matin

Copyright (c) 2016 H. Jung and I.-H. Lee. This is an open access article distributed under the Creative Commons Attribution License, which permits unrestricted use, distribution, and reproduction in any medium, provided the original work is properly cited.

\begin{abstract}
We consider device-to-device (D2D) communications in millimeter-wave (mm Wave) for the future fifth generation (5G) cellular networks. While the mm Wave systems can support multiple D2D pairs simultaneously through beamforming with highly directional antenna arrays, the $\mathrm{mm}$ Wave channel is significantly more susceptible to blockage compared to microwave; mm Wave channel studies indicate that if line-of-sight (LoS) paths are blocked, reliable mm Wave communications may not be achieved for high data-rate applications. Therefore, assuming that an outage occurs in the absence of the LoS path between two wireless devices by obstructions, we focus on connectivity of the mm Wave D2D networks. We consider two types of D2D communications: direct and indirect schemes. The connectivity performances of the two schemes are investigated in terms of (i) the probability to achieve a fully connected network $P_{\mathrm{FC}}$ and (ii) the average number of reliably connected devices $\gamma$. Through analysis and simulation, we show that, as the network size increases, $P_{\mathrm{FC}}$ and $\gamma$ decrease. Also, $P_{\mathrm{FC}}$ and $\gamma$ decrease, when the blockage parameter increases. Moreover, simulation results indicate that the hybrid direct and indirect scheme can improve both $P_{\mathrm{FC}}$ and $\gamma$ up to about 35\% compared to the nonhybrid scheme.
\end{abstract}

\section{Introduction}

With rapidly growing volume of mobile devices, the demand for capacity in mobile broadband communications increases dramatically. As a result, wireless industry is required to seek greater capacity and find new wireless spectrum beyond fourth generation $(4 \mathrm{G})$ standards. For this reason, fifth generation $(5 G)$ is envisioned to have significantly greater spectrum allocations at millimeter-wave (mm Wave) frequency bands, highly directional antenna arrays, larger coverage area, lower infrastructure costs, and higher aggregate capacity for many simultaneous users $[1,2]$. While microwave communication systems suffer from the limited spectrum, the bandwidths of several gigahertz could be available at $\mathrm{mm}$ Wave frequencies for $5 \mathrm{G}$ communication systems.

Moreover, instead of the complete control at the infrastructure side, 5G systems will exploit intelligence at the device side within different layers of the protocol stack by allowing device-to-device (D2D) connectivity [3]. The direct communications between nearby mobile devices can provide higher spectrum utilization, enhanced throughput, and better energy efficiency, while facilitating new peerto-peer applications and location-based services such as 3GPP Proximity Services (ProSe) [4] and IEEE 802.15 PeerAware Communications (PAC) [5]. Along the lines of the increasing demand of such high-rate local services, local D2D communications have been studied as underlay to Long Term Evolution-Advanced (LTE-A) 4G cellular networks [6]. As highlighted in $[6,7]$, the main challenge of a D2D-enabled air interface for the cellular networks is how to share wireless resources between cellular and D2D communications. For instance, the local D2D communications should use orthogonal channels or opportunistically access the spectrum occupied by cellular communications.

In this context, we consider D2D communications in the $\mathrm{mm}$ Wave frequency bands for the future cellular network, because the mm Wave can aid a resource sharing scheme between the D2D and cellular communications, which supports 
noninterfering concurrent links. To be specific, directional antenna arrays in $\mathrm{mm}$ Wave can reduce cochannel interference and improve spatial reuse of communication systems through large beamforming gain. This characteristic of $\mathrm{mm}$ Wave systems is also desirable to guarantee connectivity between a huge number of devices in the future wireless networks [8]. In other words, thanks to highly directional beam in mm Wave, a user equipment (UE) can communicate with another UE in proximity over a D2D link, which enables multiple D2D pairs to use the same radio resources simultaneously.

However, compared to microwave, an mm Wave communication channel experiences higher path-loss and is susceptible to blockage such as walls, trees, or even human bodies, as revealed in $[2,9,10]$. Hence, the mm Wave communication channel is a nearly bimodal channel depending on the existence of line-of-sight (LoS) path [11]. Based on this property of the mm Wave propagation, a new channel model is introduced in [12], which characterizes large-scale blockage effects using random shape theory [13]. The model proposed in [12] shows that the probability of a blockage event between two radios increases exponentially, as the separation between the two increases. This propagation model has been applied to analyze various wireless communication systems using mm Wave. The author of [14] derives the outage probability improved by macrodiversity with multiple base stations (BSs), assuming the outage event occurs when the LoS path is blocked. The authors in [15] analyze the coverage and rate performance in $\mathrm{mm}$ Wave cellular networks. Moreover, [16] presents the outage performance of the $\mathrm{mm}$ Wave wireless backhaul link between a 5G macro base station (MBS) and small-cell base stations (SBSs). Assuming Poisson point process (PPP) on the plane, the stochastic geometry used in these studies is known to be an effective tool to evaluate system performances in the cellular networks [17].

In this paper, based on the framework in $[12,14]$, we consider D2D connectivity in mm Wave networks. Reliable $\mathrm{D} 2 \mathrm{D}$ connections are required for $\mathrm{D} 2 \mathrm{D}$ data transmissions as well as cellular data offloading onto D2D connections, which can provide considerable wireless capacity gains [18]. In $\mathrm{mm}$ Wave 5G cellular networks, two kinds of D2D communications can be enabled: direct communications between two wireless devices in proximity and indirect communications, which connect two devices through base station(s) [8]. In this paper, we investigate both types of the D2D communications assuming a network consisting of one $\mathrm{mm}$ Wave BS and multiple wireless devices distributed according to a twodimensional Poisson point process (PPP).

The contributions of this paper are fourfold. First, we derive the probability distribution, mean, and variance of the interdevice distance (i.e., the distance between two randomly located devices). Second, we derive the probability that the D2D network is fully connected, which means that all the wireless devices have reliable communication links to each other [19]. Third, we also quantify the D2D connectivity in terms of the average number of reliable connections (or communication links) both for the direct and indirect D2D communication systems. Lastly, we consider a hybrid scheme, in which both the direct and indirect communications can be selectively used and present the simulation results to compare the performances of the direct and indirect schemes.

This paper is organized as follows. The system model is introduced in Section 2. The direct and indirect D2D communication systems are analyzed in Sections 3 and 4, respectively. Numerical results are presented in Section 5 . Conclusions are provided in Section 6.

\section{System Model}

We consider a 5G cellular network enabled with D2D communications with two tiers: the cell tier and the device tier. The conventional cellular communications are supported by the cell (macro or small-cell) tier, while D2D communications are covered by the device tier. The BS may have a full or partial control over the D2D communications depending on the system architecture to establish and manage the D2D links. In this paper, we focus on the connectivity of the D2D links, which indicates the potential extent of cellular data offloading onto D2D connections. Thus, the analysis in this paper does not depend on specific design aspects of the network architecture. Based on [12], we focus on outdoor environments.

We assume mm Wave 5G communication systems as shown in Figures 1(a) and 1(b), which illustrate direct and indirect D2D communications, respectively. In both cases, we assume $N$ devices are uniformly distributed over Area $\mathcal{S}$ (i.e., the area of the gray circle with radius $R$ ) with intensity $\lambda$, which means the average number of devices per unit area (i.e., devices $/ \mathrm{m}^{2}$ ), according to homogeneous PPP [20]. We assume a quasi-stationary scenario, where the network topology is stationary while D2D communications are performed.

For the indirect D2D communications in Figure 1(b), at the center of the circle, there exists a single $\mathrm{mm}$ Wave BS, which can be a small-cell BS with low power and low cost in heterogeneous networks. We note that we do not consider indirect (multihop) communications via devices as relays, because of the complexity to build a multihop route with directional antennas in the mobile scenario. In both types of D2D networks, the number of the devices in the network is assumed to be $N$, which is a random variable following Poisson probability distribution as $P_{N}(n)=$ $\left(\left(\lambda \pi R^{2}\right)^{n} / n !\right) e^{-\lambda \pi R^{2}}$, where $P_{N}(n)$ is the probability that there are $n$ devices in Area $\mathcal{S}$, and $n$ is a nonnegative integer. Therefore, the average number of the devices is given by $\mathbb{E}\{N\}=\lambda \pi R^{2}$, where $\mathbb{E}\{\cdot\}$ is the expectation operator.

As in [15], for analytical tractability, we assume the sectored antenna model, which characterizes key features of an antenna pattern. As noted in [8], the interference issue by concurrent cochannel links with omnidirectional antennas is of little concern in the $\mathrm{mm}$ Wave communications with highly directional antenna arrays. We assume that directional beamforming is performed at devices as well as the $\mathrm{mm}$ Wave BS. We note a half-duplex system is assumed. Therefore, instead of the interference-limited performance metric 


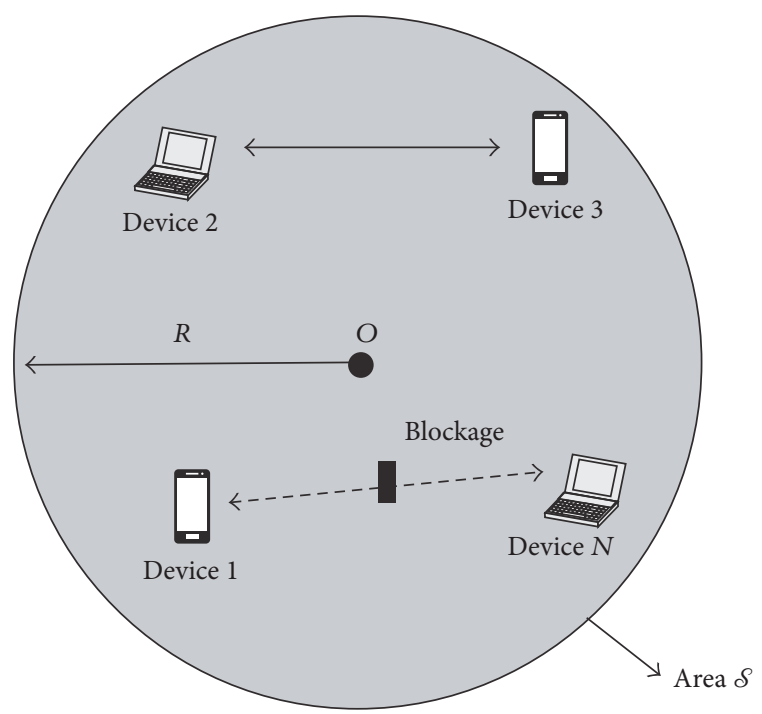

(a)

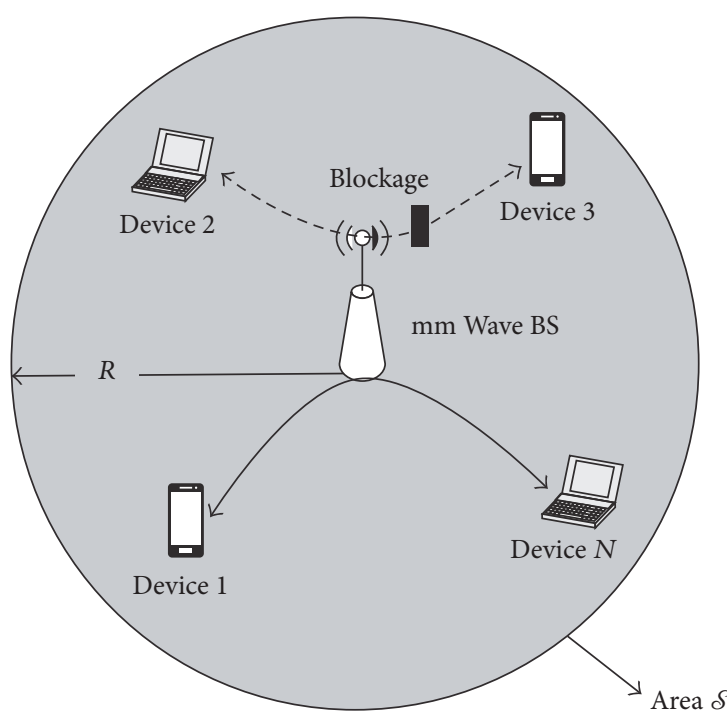

(b)

Figure 1: Two types of mm Wave D2D communications: (a) direct D2D communications and (b) indirect D2D communications.

(e.g., signal-to-noise-plus-interference ratio), the blockage problem of line-of-sight (LoS) paths may induce an outage event in mm Wave communications, especially for highspeed data transfer in multimedia or interactive applications. As in $[12,14,16]$, blockages are assumed to be impenetrable. Also, following $[12,14,16]$, we define an outage event as the case that the LoS path is blocked by obstacles.

In case of the direct D2D communications, suppose $d_{i, j}$ is the distance between devices $i$ and $j$. As in [12, 14-16], the probability of no blockage in the LoS path between the two devices is $P_{\mathrm{LoS}: i, j}=e^{-\beta d_{i, j}}$, where $\beta$ is the parameter that captures density and size of obstacles, which cause an outage due to blockage. The greater $\beta$ means obstacles with higher density and larger sizes, which results in lower $P_{\text {LoS:i,j }}$ [12]. In this paper, we assume $P_{\text {Los:i,j }}$ can be interpreted as the probability that the communication link between devices $i$ and $j$ is reliable. In other words, if the LoS path is blockage-free, we declare the corresponding communication (or connection) is reliable.

As in $[12,14,15]$, for analytical tractability, we assume the blockage events on different $\mathrm{mm}$ Wave links are mutually independent. For example, if there are three wireless terminals 1, 2, and 3, where they can be either the $\mathrm{mm}$ Wave BS or a device, the three possible communication links (i.e., 1-2, 1-3, and 2-3) have independent outage events. In general, depending on the location of wireless terminals, blockage events are not independent. For example, if the angle between two links with a common end-point or startpoint (e.g., 1-2 and 1-3) is narrow enough, the two D2D links might experience the same blockage effect. Therefore, the probability of a fully connected D2D network derived in this paper may be an upper bound as the references of system design and analysis. Moreover, we note that the numerical results in [12] show that the error caused by the independent link assumption is minor and acceptable in accuracy.

\section{Direct D2D Communications}

In this section, we consider direct D2D communications, which do not require infrastructure such as BSs. With directional antenna arrays in $\mathrm{mm}$ Wave, noninterfering concurrent $\mathrm{D} 2 \mathrm{D}$ pairs are able to share radio resources, which significantly enhances network capacity. Assuming the spatially uniform distribution of the devices, we will explore various aspects of the direct D2D communications.

3.1. Probability Distribution of $d_{i, j}$. To investigate the performance of the $\mathrm{mm}$ Wave communications between two devices, we first consider the probability distribution function (PDF) of $d_{i, j}$, since $P_{\mathrm{Los}: i, j}$ is a function of $d_{i, j}$, which is a random variable. Using Crofton's fixed point theorem in [21], we derive the PDF of $d_{i, j}$. Fixed point theorem permits the evaluation of some definite integrals without directly performing the integrations, which is especially useful to derive geometric probability distributions. Suppose that there are $n$ points $\xi_{1}, \xi_{2}, \ldots, \xi_{n}$, which are randomly distributed on a domain $\mathcal{S}$. Let $Q$ be an event that depends on the position of the $n$ points, and let $d S$ be an infinitesimal boundary of $\mathcal{S}$. Then, Crofton's fixed point theorem gives the following formula:

$$
d \operatorname{Pr}[Q]=\frac{n\left(\operatorname{Pr}\left[Q \mid \xi_{1} \in d \mathcal{S}\right]-\operatorname{Pr}[Q]\right)}{\mathcal{S}} d \mathcal{S}
$$

where $\operatorname{Pr}\left[Q \mid \xi_{1} \in d \delta\right]$ is the probability that $Q$ occurs when one of the random points $\xi_{1}$ is on the boundary $d \mathcal{S}$ of $\mathcal{S}$.

To derive the PDF of $d_{i, j}$, suppose that $Q$ is the event that two points (i.e., $n=2$ ) in the circle with the radius $R$ (i.e., $\mathcal{S}=\pi R^{2}$ ) are separated by a distance between $x$ and $x+d x$, as shown in Figure 2. Also, let $C$ be the event that one point is on the circumference $d \mathcal{S}$. The probability of the event $Q$ is 


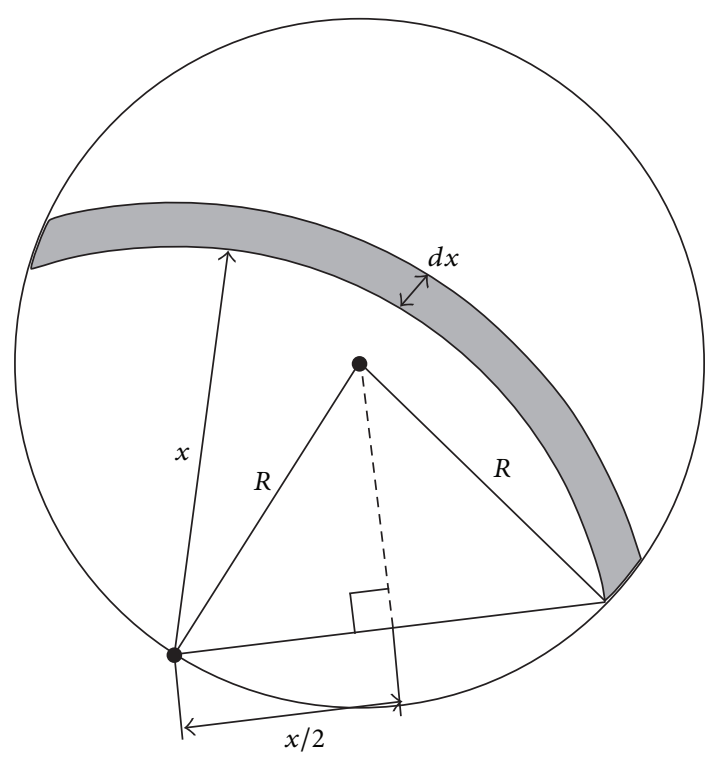

FIgURE 2: Illustration to derive the PDF of $d_{i, j}$.

denoted by $\operatorname{Pr}[Q]$, and the conditional probability of $Q$ given $C$ is denoted by $\operatorname{Pr}[Q \mid C]$. From (1), we have

$$
\begin{aligned}
d \operatorname{Pr}[Q] & =\frac{2(\operatorname{Pr}[Q \mid C]-\operatorname{Pr}[Q])}{\mathcal{S}} d \mathcal{S} \\
& =\frac{2(\operatorname{Pr}[Q \mid C]-\operatorname{Pr}[Q])}{\pi R^{2}} 2 \pi R d R \\
& =\frac{4(\operatorname{Pr}[Q \mid C]-\operatorname{Pr}[Q])}{R} d R,
\end{aligned}
$$

where $\operatorname{Pr}[Q \mid C]$ is given by

$$
\operatorname{Pr}[Q \mid C]=\frac{2 x d x \cos ^{-1}(x / 2 R)}{\pi R^{2}} .
$$

Therefore, when plugging (3) into (2), it gives

$$
R d \operatorname{Pr}[Q]+4 \operatorname{Pr}[Q] d R=\frac{8 x d x \cos ^{-1}(x / 2 R)}{\pi R^{2}} d R .
$$

Multiplying both sides by $R^{3}$, we obtain

$$
\begin{aligned}
R^{4} d & \operatorname{Pr}[Q]+4 R^{3} \operatorname{Pr}[Q] d R \\
\quad= & \frac{8 R x d x \cos ^{-1}(x / 2 R)}{\pi} d R .
\end{aligned}
$$

Thus, if we integrate both sides, it gives

$$
\begin{aligned}
& R^{4} \operatorname{Pr}[Q]=\frac{4 x^{2} d x}{\pi} \int \frac{2 R}{x} \cos ^{-1}\left(\frac{x}{2 R}\right) d R \\
& =\frac{x d x}{\pi}\left(4 R^{2} \cos ^{-1}\left(\frac{x}{2 R}\right)-2 x R \sqrt{1-\frac{x^{2}}{4 R^{2}}}\right)+K,
\end{aligned}
$$

where the constant $K=0$, because $\operatorname{Pr}[Q]=0$ when $R=x / 2$. Hence, the PDF of $d_{i, j}$ is expressed as

$$
\begin{aligned}
f_{d_{i, j}}(x) & =\frac{\operatorname{Pr}[Q]}{d x} \\
& =\frac{2 x}{\pi R^{2}}\left(2 \cos ^{-1}\left(\frac{x}{2 R}\right)-\frac{x}{R} \sqrt{1-\frac{x^{2}}{4 R^{2}}}\right),
\end{aligned}
$$

where $0<x<2 R$. The derived PDF in (7) is the same as [22]. The mean and variance of $d_{i, j}$ are given by $\mathbb{E}\left\{d_{i, j}\right\}=128 R / 45 \pi$ and $\operatorname{VAR}\left\{d_{i, j}\right\}=R^{2}-(128 R / 45 \pi)^{2}$, respectively.

3.2. Fully Connected Network via Direct D2D Communications. In this section, we derive the probability that all the wireless devices are interconnected via direct D2D communications in $\mathrm{mm}$ Wave. Suppose a Bernoulli random variable $U_{i, j}$ is defined as

$$
U_{i, j}= \begin{cases}1, & \text { w.p. } P_{\mathrm{LoS}: i, j}=\exp \left(-\beta d_{i, j}\right), \\ 0, & \text { w.p. } 1-P_{\mathrm{LoS}: i, j}=1-\exp \left(-\beta d_{i, j}\right),\end{cases}
$$

where if $U_{i, j}=1$, it means that the $\mathrm{mm}$ Wave link between devices $i$ and $j$ is reliable. In other words, following assumption of impenetrable blockage in $[12,14]$, we declare an outage event of the direct D2D transmission between devices $i$ and $j$, when $U_{i, j}=0$. If there are $N(\geq 2)$ devices, there can exist $\left(\begin{array}{c}N \\ 2\end{array}\right)=N(N-1) / 2$ possible $\mathrm{mm}$ Wave links between any two devices as in [19] (with $N$ devices or nodes in a network, the maximum number of concurrent "active" D2D links (or pairs) is $N / 2$. However, we consider all the possible link combinations among $N$ devices $\left(\begin{array}{c}N \\ 2\end{array}\right)=N(N-1) / 2$ to check if the network is fully connected). When all of the $N(N-1) / 2$ direct D2D links are reliable, we assume that the network is fully connected [19], which can be regarded as a fully meshed network [23].

Therefore, the probability to achieve the fully connected network can be represented as

$$
P_{\mathrm{FC}}=\frac{\sum_{n=2}^{\infty} \operatorname{Pr}\left[\text { all } U_{i, j} \text { 's are one } \mid N=n\right] \operatorname{Pr}[N=n]}{\operatorname{Pr}[N \geq 2]},
$$

where $1 \leq i, j \leq n$, where $i \neq j$. With the independent link assumption as in $[12,14]$, the conditional probability in (9) can be simplified as

$$
\operatorname{Pr}\left[\text { all } U_{i, j} \text { 's are one } \mid N=n\right]=\left(\mathbb{E}\left\{e^{-\beta d_{i, j}}\right\}\right)^{n(n-1) / 2},
$$

where the distance $d_{i, j}$ between devices $i$ and $j$ follows the PDF in (7). Suppose $\mathscr{A}=\mathbb{E}\left\{e^{-\beta d_{i j}}\right\}$, which can be calculated by Taylor expansion as follows:

$$
\begin{aligned}
\mathscr{A} & =\int_{0}^{2 R} e^{-\beta x} f_{d_{i, j}}(x) d x \\
& \approx\left[1+\frac{\beta^{2}}{2}\left(R^{2}-\left(\frac{128 R}{45 \pi}\right)^{2}\right)\right] \exp \left(-\beta \frac{128 R}{45 \pi}\right) .
\end{aligned}
$$


Thus, $P_{\mathrm{FC}}$ in (9) can be simplified as

$$
\begin{aligned}
P_{\mathrm{FC}} & =\mathbb{E}\left\{\mathscr{A}^{N(N-1) / 2} \mid N \geq 2\right\} \\
& =\frac{\sum_{n=2}^{\infty} \mathscr{A}^{n(n-1) / 2} \cdot \operatorname{Pr}[N=n]}{\operatorname{Pr}[N \geq 2]} \\
& =\frac{\sum_{n=2}^{\infty} \mathscr{A}^{n(n-1) / 2} \cdot\left(\left(\lambda \pi R^{2}\right)^{n} / n !\right) e^{-\lambda \pi R^{2}}}{1-\exp \left(-\lambda \pi R^{2}\right)\left(1+\lambda \pi R^{2}\right)} .
\end{aligned}
$$

3.3. Ratio of Reliable Direct D2D Connections. If the fully connected network cannot be achieved, we can estimate the average number of reliably connected devices through direct D2D communications. If we define a set $T_{\text {dir }}:=\left\{U_{i, j} \mid U_{i, j}=\right.$ $1\}$, its cardinality $\left|T_{\text {dir }}\right|$ is the number of the reliable direct D2D links. Thus, for $N \geq 2$, the ratio of the reliable D2D connections to all of the possible D2D pairs in the network can be derived as

$$
\begin{aligned}
\gamma_{\text {dir }} & =\frac{1}{\operatorname{Pr}[N \geq 2]} \sum_{n=2}^{\infty} \frac{\mathbb{E}\left\{\left|T_{\text {dir }}\right| \mid N=n\right\}}{n(n-1) / 2} \operatorname{Pr}[N=n] \\
& =\mathscr{A}
\end{aligned}
$$

where $\mathbb{E}\left\{\left|T_{\text {dir }}\right| \mid N=n\right\}$ is in fact the mean of binomial distribution $B(n(n-1) / 2, p=\mathscr{A})$, which gives $\mathbb{E}\left\{\left|T_{\text {dir }}\right| \mid N=\right.$ $n\}=\mathscr{A} n(n-1) / 2$. As a result, $\gamma_{\text {dir }}=\mathscr{A}$, and it is not a function of the device density $\lambda$.

3.4. Impacts of System Parameters. In this section, we consider the impacts of the network size $R$ and the blockage parameter $\beta$ on $P_{\mathrm{FC}}$ and $\gamma_{\mathrm{dir}}$. The analysis in this section will be verified by comparing with the simulation results in Section 5 .

3.4.1. Radius $R$. To analyze the impact of the network size indicated by $R$, we first need to investigate the derivative of $\mathscr{A}$ in (11) in terms of $R$ as below:

$$
\begin{aligned}
\frac{d \mathscr{A}}{d R} & =-\frac{\beta e^{-128 \beta R / 45 \pi}}{91125 \pi^{3}}\left[\left(8 \beta \sqrt{2025 \pi^{2}-16384} R\right.\right. \\
& \left.+\frac{737280 \pi-91125 \pi^{3}}{16 \sqrt{2025 \pi^{2}-16384}}\right)^{2} 259200 \pi^{2} \\
& \left.-\left(\frac{737280 \pi-91125 \pi^{3}}{16 \sqrt{2025 \pi^{2}-16384}}\right)^{2}\right],
\end{aligned}
$$

which satisfies $d \mathscr{A} / d R<0$ for any $\beta$ and $R$. Therefore, $\gamma_{\text {dir }}=$ $\mathscr{A}$ is a decreasing function of $R$. Moreover, we can find that $P_{\mathrm{FC}} \rightarrow 0$ as $R \rightarrow \infty$.

3.4.2. Blockage Parameter $\beta$. As in the previous section regarding $R$, we find the derivative of $\mathscr{A}$ in terms of $\beta$ to investigate the impact of $\beta$ as

$$
\frac{d \mathscr{A}}{d \beta}=-\frac{R e^{-128 \beta R / 45 \pi}}{91125 \pi^{3}}\left[\left(8 R \sqrt{2025 \pi^{2}-16384} \beta\right.\right.
$$

$$
\begin{aligned}
& \left.+\frac{737280 \pi-91125 \pi^{3}}{16 \sqrt{2025 \pi^{2}-16384}}\right)^{2} 259200 \pi^{2} \\
& \left.-\left(\frac{737280 \pi-91125 \pi^{3}}{16 \sqrt{2025 \pi^{2}-16384}}\right)^{2}\right]
\end{aligned}
$$

which satisfies $d \mathscr{A} / d \beta<0$ for any $\beta$ and $R$. Therefore, $\gamma_{\text {dir }}=$ $\mathscr{A}$ is a decreasing function of $\beta$. Also, when $\beta \rightarrow 0, \mathscr{A} \rightarrow 1$. Consequently, $P_{\mathrm{FC}} \rightarrow 1$ as $\beta \rightarrow 0$. On the other hand, as $\beta \rightarrow \infty, \mathscr{A} \rightarrow 0$, which results in $P_{\mathrm{FC}} \rightarrow 0$.

\section{Indirect D2D Communications via mm Wave Base Station}

In this section, we investigate indirect D2D communications hopping via the $\mathrm{mm}$ Wave $\mathrm{BS}$, where it is assumed that direct D2D links are unavailable. If we have an indirect D2D communication pair, two consecutive LoS links "from one device to the mm Wave BS" and "from the mm Wave BS to the other device" are required to be reliable.

4.1. Fully Connected Network via Indirect D2D Communications. For the indirect D2D communication from devices $i$ to $j$ via the BS, both "device $i$ to BS" and "BS to device $j$ " links should have blockage-free LoS paths. Therefore, to have a fully connected network with $N \geq 2$ hopping over the BS, all the LoS links between the mm Wave BS and $N$ devices should not have blockage. As in the previous section, suppose a Bernoulli random variable $W_{i}$ is defined as

$$
W_{i}= \begin{cases}1, & \text { w.p. } P_{\mathrm{LoS}: i}=\exp \left(-\beta r_{i}\right) \\ 0, & \text { w.p. } 1-P_{\mathrm{LoS}: i}=1-\exp \left(-\beta r_{i}\right)\end{cases}
$$

where if $W_{i}=1$, the mm Wave communication between the mm Wave BS and device $i$ is successful.

Also, $r_{i}$ is the distance between the mm Wave BS and device $i$, which follows the PDF of $f_{r_{i}}(x)=2 x / R^{2}$ for $0 \leq$ $x \leq R$. Therefore, the probability to create a fully connected network is given by

$$
\begin{aligned}
P_{\mathrm{FC}} & =\frac{\sum_{n=2}^{\infty} \operatorname{Pr}\left[\text { all } W_{i} \text { 's are one } \mid N=n\right] \operatorname{Pr}[N=n]}{\operatorname{Pr}[N \geq 2]} \\
& =\frac{\sum_{n=2}^{\infty}\left(\mathbb{E}\left\{e^{-\beta r_{i}}\right\}\right)^{n} \operatorname{Pr}[N=n]}{\operatorname{Pr}[N \geq 2]},
\end{aligned}
$$

where $1 \leq i \leq n$. If $\mathscr{B}=\mathbb{E}\left\{e^{-\beta r_{i}}\right\}$, it gives

$$
\mathscr{B}=\int_{0}^{R} e^{-\beta x} f_{r_{i}}(x) d x=\frac{2}{\beta R^{2}}\left[\frac{1}{\beta}-\left(R+\frac{1}{\beta}\right) e^{-\beta R}\right] .
$$


Hence, $P_{\mathrm{FC}}$ is obtained as

$$
\begin{aligned}
P_{\mathrm{FC}} & =\frac{\sum_{n=2}^{\infty} \mathscr{B}^{n} \operatorname{Pr}[N=n]}{\operatorname{Pr}[N \geq 2]} \\
& =\sum_{n=2}^{\infty} \frac{\mathscr{B}^{n}\left(\lambda \pi R^{2}\right)^{n} \cdot e^{-\lambda \pi R^{2}}}{\operatorname{Pr}[N \geq 2] \cdot n !} \\
& =\frac{e^{\mathscr{B} \lambda \pi R^{2}}-\mathscr{B} \lambda \pi R^{2}-1}{e^{\lambda \pi R^{2}}-\lambda \pi R^{2}-1} .
\end{aligned}
$$

4.2. Ratio of Reliable Indirect D2D Connections. If we define a set $T_{\text {ind }}:=\left\{W_{i} \mid W_{i}=1\right\}$, its cardinality $\left|T_{\text {ind }}\right|$ is the number of reliable "device to the mm Wave BS" links. Because any devices connected to the $\mathrm{mm}$ Wave BS can indirectly communicate with each other, for $\left|T_{\text {ind }}\right| \geq 2$, there are $\left|T_{\text {ind }}\right|\left(\left|T_{\text {ind }}\right|-1\right) / 2$ reliable $\mathrm{D} 2 \mathrm{D}$ connections. For a given $N=$ $n \geq 2,\left|T_{\text {ind }}\right|$ follows a binomial distribution $B(n, p=\mathscr{B})$, where $\mathscr{B}$ is derived in (18). Therefore, the ratio of the reliable indirect $\mathrm{D} 2 \mathrm{D}$ connections is given by

$$
\begin{aligned}
\gamma_{\text {ind }} & =\frac{1}{\operatorname{Pr}[N \geq 2]} \\
& \cdot \sum_{n=2}^{\infty} \frac{\mathbb{E}\left\{\left|T_{\text {ind }}\right|\left(\left|T_{\text {ind }}\right|-1\right) / 2 \mid N=n\right\} \cdot \operatorname{Pr}[N=n]}{n(n-1) / 2} \\
& =\frac{1}{\operatorname{Pr}[N \geq 2]} \\
& \cdot \sum_{n=2}^{\infty} \frac{\left(\mathbb{E}\left\{\left|T_{\text {ind }}\right|^{2} \mid N=n\right\}-\mathbb{E}\left\{\left|T_{\text {ind }}\right| \mid N=n\right\}\right) / 2}{n(n-1) / 2} \\
& \cdot \operatorname{Pr}[N=n]=\frac{1}{\operatorname{Pr}[N \geq 2]} \sum_{n=2}^{\infty} \frac{n^{2} \mathscr{B}^{2}-n \mathscr{B}^{2}}{n(n-1)} \\
& \cdot \operatorname{Pr}[N=n]=\mathscr{B}^{2} .
\end{aligned}
$$

As $\gamma_{\text {dir }}$ in (13), $\gamma_{\text {ind }}$ is not a function of the device density $\lambda$, either.

4.3. Impacts of System Parameters. As in the previous section about the direct D2D communications, we analyze the impacts of $R$ and $\beta$ on $P_{\mathrm{FC}}$ and $\gamma_{\mathrm{dir}}$.

4.3.1. Radius $R$. We first consider how $\mathscr{B}$ changes as $R$ varies by considering two extreme cases. For $R \rightarrow 0$ and $\infty$, we have

$$
\begin{aligned}
& \lim _{R \rightarrow 0} \mathscr{B}=\lim _{R \rightarrow 0} \frac{1 / \beta-e^{-\beta R}(1 / \beta+R)}{\beta R}=\frac{\beta}{\beta}=1, \\
& \lim _{R \rightarrow \infty} \mathscr{B}=\lim _{R \rightarrow 0} \frac{2}{\beta^{2} R^{2}}=0 .
\end{aligned}
$$

Thus, $\gamma_{\text {ind }}=\mathscr{B}^{2} \rightarrow 1$ as $R \rightarrow 0$. On the other hand, $\gamma_{\text {ind }}=$ $\mathscr{B}^{2} \rightarrow 0$ as $R \rightarrow \infty$. Furthermore, $\lim _{R \rightarrow 0} P_{\mathrm{FC}}=\lim _{R \rightarrow 0} \mathscr{B}^{2}=$ 1 . In contrast, $P_{\mathrm{FC}} \rightarrow 0$ as $R \rightarrow \infty$.

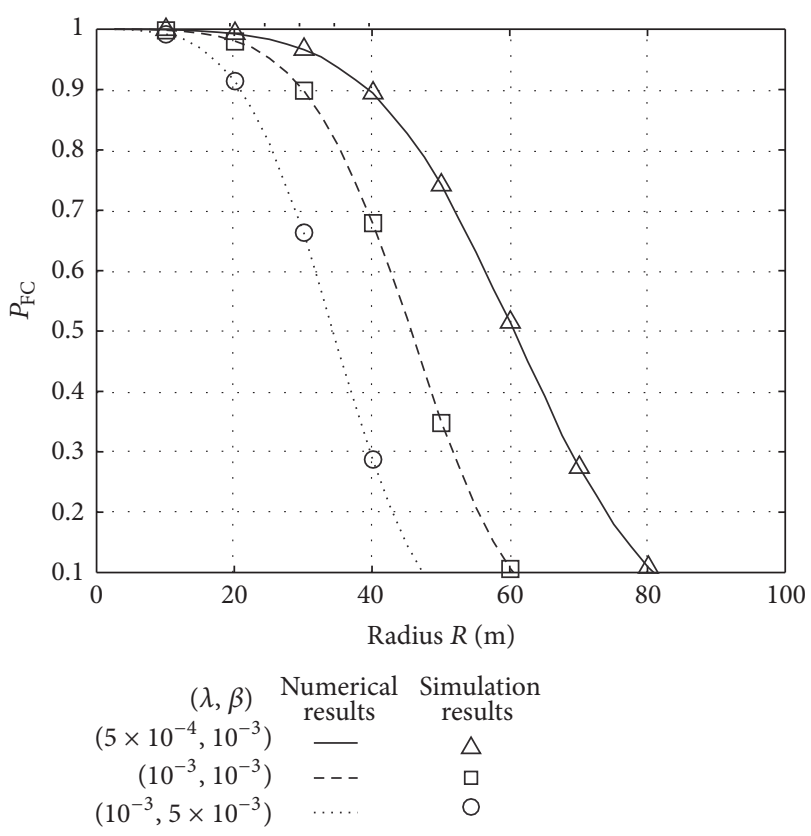

Figure 3: Direct D2D: $P_{\mathrm{FC}}$ versus $R$ with $\lambda=\{0.001,0.0005\}$ and $\beta=\{0.001,0.005\}$.

4.3.2. Blockage Parameter $\beta$. We can readily find that $\mathscr{B} \rightarrow 1$ and 0 , as $\beta \rightarrow 0$ and $\infty$, respectively. Therefore, $\gamma_{\text {ind }}=\mathscr{B}^{2}$ has the same limiting values. Also, it gives $P_{\mathrm{FC}} \rightarrow 1$ and 0 , as $\beta \rightarrow 0$ and $\infty$, respectively.

\section{Numerical Results}

In this section, we present numerical results of the mm Wave D2D communication systems via both the direct and indirect communications.

5.1. Direct D2D Communications. Figure 3 shows the probability to achieve a fully connected D2D network using the direct D2D communications. The horizontal axis $R$ represents the radius of Area $\mathcal{S}$ in Figure 1(a). The solid, dashed, and dotted lines represent $P_{\mathrm{FC}}$ in (12) obtained numerically with different combinations of $\lambda$ and $\beta$. Moreover, the symbols indicate the corresponding Monte Carlo simulation results, which are consistent with the numerical results. All the three graphs decrease, as the radius $R$ increases. Also, comparing the solid and dashed lines $(\lambda=0.0005$ and 0.001 with the same $\beta$ ), the higher $\lambda$ gives the lower likelihood of the fully connected network. Similarly, as $\beta$ increases (the dashed and dotted lines), $P_{\mathrm{FC}}$ decreases. In other words, the probability $P_{\mathrm{FC}}$ is a decreasing function of both the device density $\lambda$ and the blockage parameter $\beta$.

Figure 4 shows the ratio of the reliable direct D2D connections $\gamma_{\text {dir }}$ in (13). The three curves denoted by the solid, dashed, and dotted lines correspond to $\beta=\{0.001,0.01,0.1\}$, respectively. Also, the simulation results indicated by the symbols are in line with the numerical results. As $R$ increases, $\gamma_{\text {dir }}$ decreases. Furthermore, the ratio of the reliable D2D connections decreases, as $\beta$ increases, because of higher blockage 


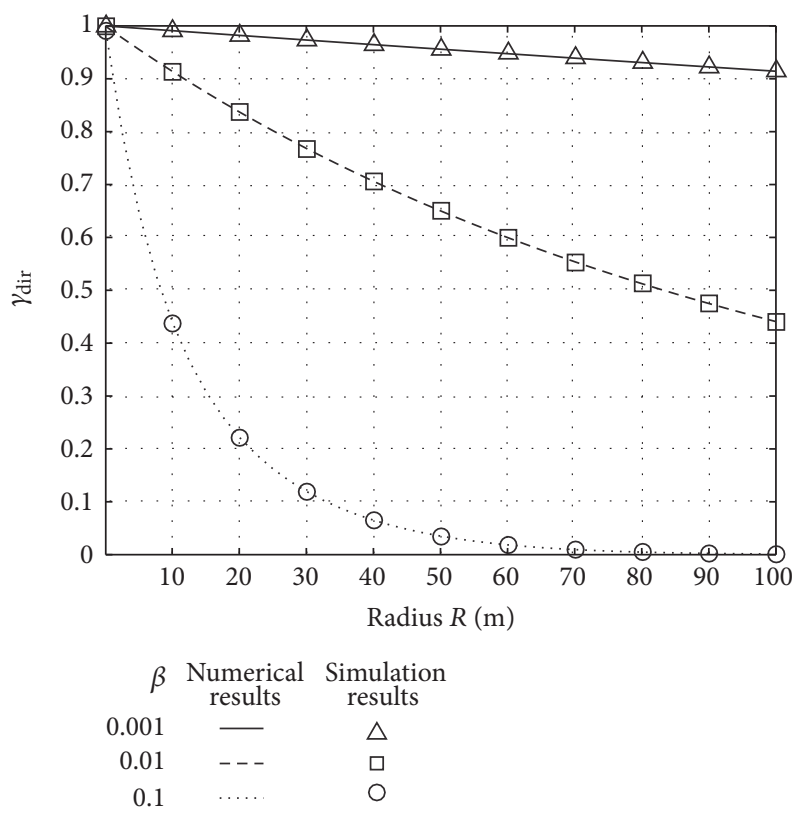

FIgURE 4: Direct D2D: $\gamma_{\text {dir }}=\mathscr{A}$ versus $R$ with $\beta=\{0.001,0.01,0.1\}$.

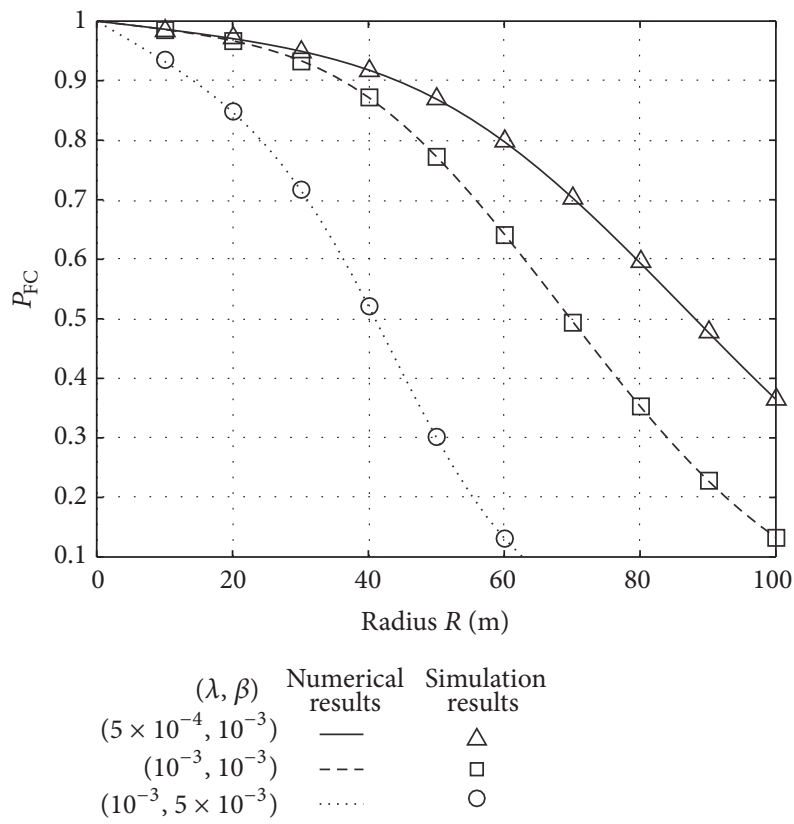

FIgURe 5: Indirect D2D: $P_{\mathrm{FC}}$ versus $R$ with $\lambda=\{0.001,0.0005\}$ and $\beta=\{0.001,0.005\}$.

effects. These observations about the system parameters $R$ and $\beta$ are consistent with our analysis in Section 3.4.

5.2. Indirect $D 2 D$ Communications. Figure 5 shows the results of $P_{\mathrm{FC}}$ for the indirect $\mathrm{D} 2 \mathrm{D}$ communications in (19). The three (solid, dashed, and dotted) lines indicate different $\lambda$ and $\beta$ combinations, while the symbols represent the corresponding simulation results. As in the direct D2D communication case, the probability $P_{\mathrm{FC}}$ decreases, as $R$

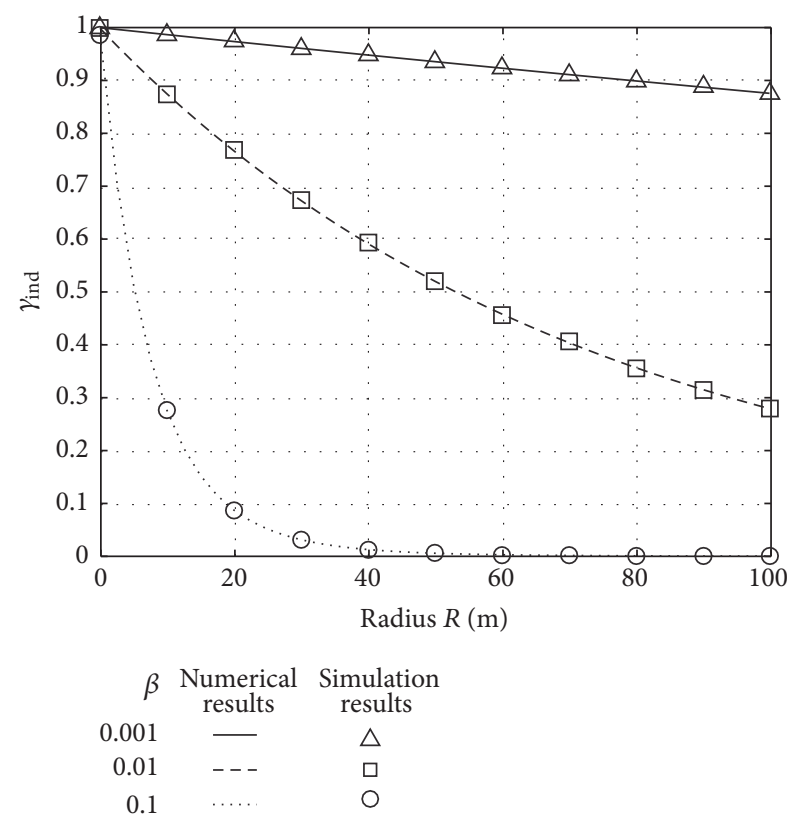

FIGURE 6: Indirect D2D: $\gamma_{\text {ind }}=\mathscr{B}^{2}$ versus $R$ with $\beta=\{0.001,0.01$, $0.1\}$.

increases. We can observe that $P_{\mathrm{FC}}$ is a decreasing function of $\lambda$. Also, $P_{\mathrm{FC}}$ decreases, as $\beta$ increases. The same trends can be observed in Figure 6, which is in line with our analysis in Section 4.3.

Compared to the direct communication case, for the same parameters $\lambda, \beta$, and $R, P_{\mathrm{FC}}$ is significantly higher with the $\mathrm{D} 2 \mathrm{D}$ communication hopping via the $\mathrm{mm}$ Wave $\mathrm{BS}$, because the possible distance of the indirect D2D links, that is, $0 \leq$ $r_{i} \leq R$, is shorter than that of the direct D2D links; that is, $0 \leq d_{i, j} \leq 2 R$. Also, for the average separation between two terminals (which are one device and the mm Wave BS for the indirect D2D, but two devices in the direct D2D transmissions), $\mathbb{E}\left\{d_{i, j}\right\} \approx 0.91 R>\mathbb{E}\left\{r_{i}\right\} \approx 0.67 R$, which implies a higher probability of blockage in the direct D2D compared to the indirect D2D communications.

In contrast, when it comes to the average ratios of the reliable D2D connections, $\gamma_{\text {ind }}$ is smaller than $\gamma_{\text {dir }}$. This can be explained by comparing the exponential terms, which dominate the polynomial terms for large enough $\beta$ and $R$, of the two ratios as

$$
\begin{aligned}
& \gamma_{\text {dir }}=\mathscr{A} \propto \exp \left(-\frac{128}{45 \pi} \beta R\right) \approx \exp (-0.9 \beta R), \\
& \gamma_{\text {ind }}=\mathscr{B}^{2} \propto[\exp (-\beta R)]^{2} \approx \exp (-2 \beta R) .
\end{aligned}
$$

Therefore, for large enough $\beta$ and $R, \gamma_{\text {dir }}>\gamma_{\text {ind }}$.

5.3. Hybrid D2D Communications. In this section, we present the D2D connectivity with both the direct and indirect communications jointly exploited. In other words, we consider a hybrid scheme, where all the devices are permitted to use both direct and indirect links to communicate with each other. Therefore, for a D2D pair, we assume their D2D link 
TABLE 1: $P_{\mathrm{FC}}$ for direct, indirect, and hybrid (direct + indirect) D2D communications.

\begin{tabular}{cccccc}
\hline $\begin{array}{c}\text { Parameters } \\
(\lambda, \beta)\end{array}$ & $R$ & Direct & $\begin{array}{c}P_{\mathrm{FC}} \\
\text { Indirect }\end{array}$ & Hybrid & Change $(\%)$ \\
\hline$(0.0005,0.001)$ & 50 & 0.9992 & 0.9863 & 1.0000 & 0.08 \\
& 100 & 0.0063 & 0.3646 & 0.4828 & 32.4 \\
\hline & 10 & 0.9983 & 0.9859 & 1.0000 & 0.17 \\
$(0.001,0.001)$ & 50 & 0.3479 & 0.7715 & 0.9241 & 19.8 \\
& 100 & 0.0000 & 0.1329 & 0.1563 & 17.6 \\
\hline & 10 & 0.9913 & 0.9315 & 0.9918 & 0.05 \\
$(0.001,0.005)$ & 50 & 0.0595 & 0.2990 & 0.4026 & 34.6 \\
& 100 & 0.0000 & 0.0002 & 0.0002 & - \\
\hline
\end{tabular}

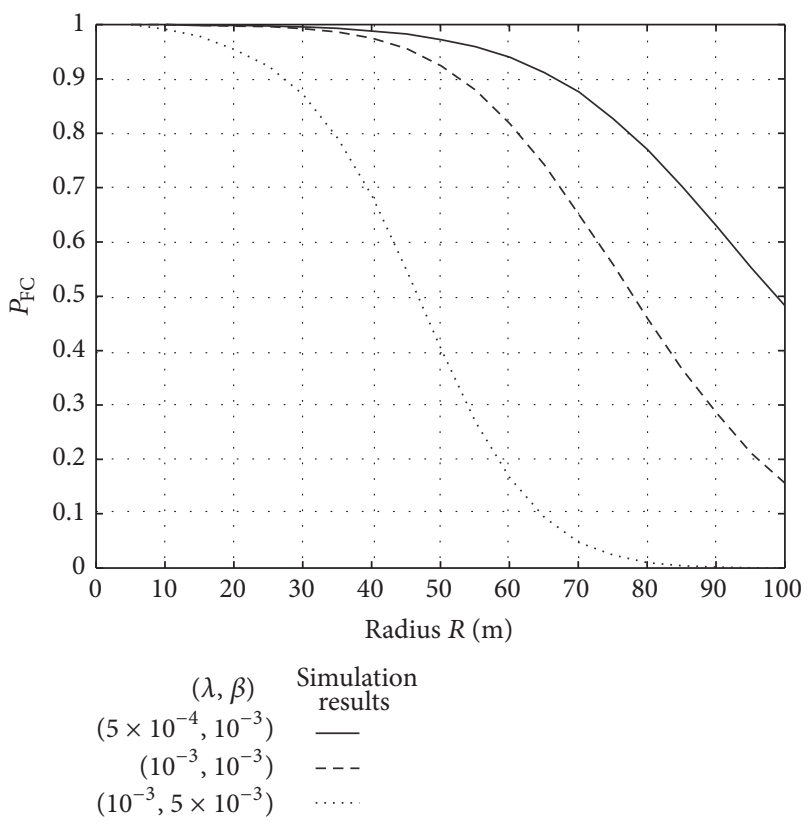

Figure 7: Hybrid D2D: $P_{\mathrm{FC}}$ versus $R$ with $\lambda=\{0.001,0.0005\}$ and $\beta=\{0.001,0.005\}$.

is reliable as long as one of the two (direct or indirect links) is reliable.

Table 1 and Figure 7 show $P_{\mathrm{FC}}$ with the hybrid (direct + indirect) D2D communications. Also, Table 2 and Figure 8 present the ratio of the reliable $\mathrm{D} 2 \mathrm{D}$ connections using the hybrid scheme. In the two tables, the last column indicates the performance improvements in percentage by using the hybrid scheme compared to the nonhybrid case assuming that we pick the one with the better performance between the direct and indirect connections. As shown in the tables and figures, when the two communication links are jointly used, both $P_{\mathrm{FC}}$ and $\gamma$ could be improved significantly. For example, in Table 1, $P_{\mathrm{FC}}$ becomes almost one with the small $R$ for the given $\lambda$ and $\beta$. Also, Table 2 shows the increase in $\gamma$ up to $35.5 \%$.
TABLE 2: $\gamma$ for direct, indirect, and hybrid (direct + indirect) D2D communications.

\begin{tabular}{cccccc}
\hline \multicolumn{2}{c}{ Parameters } & \multicolumn{4}{c}{$\gamma$} \\
$\beta$ & $R$ & Direct & Indirect & Hybrid & Change (\%) \\
\hline \multirow{3}{*}{0.001} & 10 & 0.9909 & 0.9866 & 0.9999 & 0.09 \\
& 50 & 0.9559 & 0.9355 & 0.9972 & 4.3 \\
& 100 & 0.9143 & 0.8757 & 0.9893 & 8.2 \\
\hline \multirow{3}{*}{0.01} & 10 & 0.9134 & 0.8745 & 0.9891 & 8.3 \\
& 50 & 0.6497 & 0.5201 & 0.8319 & 28.0 \\
& 100 & 0.4408 & 0.2793 & 0.5970 & 35.4 \\
\hline \multirow{3}{*}{0.1} & 10 & 0.4376 & 0.2759 & 0.5928 & 35.5 \\
& 50 & 0.0349 & 0.0059 & 0.0406 & 16.3 \\
& 100 & 0.0012 & 0.0004 & 0.0016 & 33.3 \\
\hline
\end{tabular}

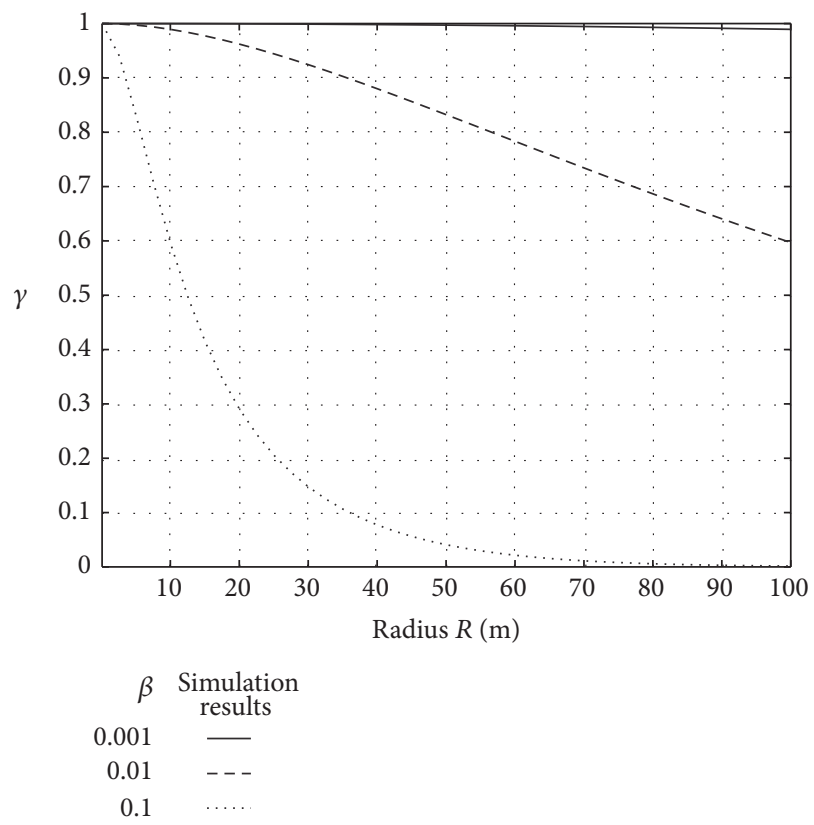

Figure 8: Hybrid D2D: $\gamma$ versus $R$ with $\beta=\{0.001,0.01,0.1\}$.

\section{Conclusion}

In this paper, we consider D2D communications in $\mathrm{mm}$ Wave, where a device can communicate with another device directly or via the mm Wave BS. Assuming uniform distribution of devices according to PPP and the blockage model, we derive the probability of the fully connected network for both direct and indirect communications. Moreover, we analyze the ratio of the reliable $\mathrm{D} 2 \mathrm{D}$ connections with the two types of communications. Through analysis and numerical results, we observe that both connectivity performance metrics decrease, as the network size and the blockage parameter increase. Also, the simulation results show that if the hybrid direct and indirect schemes are used, both connectivity performances can be enhanced up to about $35 \%$ compared to the nonhybrid case. 


\section{Competing Interests}

The authors declare that they have no competing interests.

\section{Acknowledgments}

This work was supported by the Incheon National University Research Grant in 2016.

\section{References}

[1] C.-X. Wang, F. Haider, X. Gao et al., "Cellular architecture and key technologies for 5G wireless communication networks," IEEE Communications Magazine, vol. 52, no. 2, pp. 122-130, 2014.

[2] T. S. Rappaport, S. Sun, R. Mayzus et al., "Millimeter wave mobile communications for $5 \mathrm{G}$ cellular: it will work!," IEEE Access, vol. 1, pp. 335-349, 2013.

[3] F. Boccardi, R. W. Heath, A. Lozano, T. L. Marzetta, and P. Popovski, "Five disruptive technology directions for 5G," IEEE Communications Magazine, vol. 52, no. 2, pp. 74-80, 2014.

[4] 3rd Generation Partnership Project (3GPP), "Technical specification group SA; Feasibility study for proximity services (ProSe) (Release 12)," Tech. Rep. TR 22.803 V1.0.0, 2012.

[5] IEEE 802.15.8, "Peer-Aware Communications (PAC)," http:// www.ieee802.org/15/pub/TG8.html.

[6] K. Doppler, M. Rinne, C. Wijting, C. B. Ribeiro, and K. Hug, "Device-to-device communication as an underlay to LTEadvanced networks," IEEE Communications Magazine, vol. 47, no. 12, pp. 42-49, 2009.

[7] X. Lin, J. G. Andrews, A. Ghosh, and R. Ratasuk, "An overview of 3GPP device-to-device proximity services," IEEE Communications Magazine, vol. 52, no. 4, pp. 40-48, 2014.

[8] J. Qiao, X. S. Shen, J. W. Mark, Q. Shen, Y. He, and L. Lei, "Enabling device-to-device communications in millimeterwave 5G cellular networks," IEEE Communications Magazine, vol. 53, no. 1, pp. 209-215, 2015.

[9] T. S. Rappaport, F. Gutierrez, E. Ben-Dor, J. N. Murdock, Y. Qiao, and J. I. Tamir, "Broadband millimeter-wave propagation measurements and models using adaptive-beam antennas for outdoor urban cellular communications," IEEE Transactions on Antennas and Propagation, vol. 61, no. 4, pp. 1850-1859, 2013.

[10] S. Rajagopal, S. Abu-Surra, and M. Malmirchegini, "Channel feasibility for outdoor non-line-of-sight mmwave mobile communication," in Proceedings of the 76th IEEE Vehicular Technology Conference (VTC '12), pp. 1-6, September 2012.

[11] J. G. Andrews, S. Buzzi, W. Choi et al., "What will 5G be?" IEEE Journal on Selected Areas in Communications, vol. 32, no. 6, pp. 1065-1082, 2014.

[12] T. Bai, R. Vaze, and R. W. Heath, "Analysis of blockage effects on urban cellular networks," IEEE Transactions on Wireless Communications, vol. 13, no. 9, pp. 5070-5083, 2014.

[13] D. Stoyan, W. Kendall, and J. Mecke, Geometry and Its Applications, John Wiley \& Sons, New York, NY, USA, 2nd edition, 1995.

[14] J. Choi, "On the macro diversity with multiple BSs to mitigate blockage in millimeter-wave communications," IEEE Communications Letters, vol. 18, no. 9, pp. 1623-1656, 2014.

[15] T. Bai and R. W. Heath, "Coverage and rate analysis for millimeter-wave cellular networks," IEEE Transactions on Wireless Communications, vol. 14, no. 2, pp. 1100-1114, 2015.
[16] H. Jung and I.-H. Lee, "Outage analysis of millimeter-wave wireless backhaul in the presence of blockage," IEEE Communications Letters, vol. 20, no. 11, pp. 2268-2271, 2016.

[17] J. G. Andrews, F. Baccelli, and R. K. Ganti, "A tractable approach to coverage and rate in cellular networks," IEEE Transactions on Communications, vol. 59, no. 11, pp. 3122-3134, 2011.

[18] S. Andreev, O. Galinina, A. Pyattaev, K. Johnsson, and Y. Koucheryavy, "Analyzing assisted offloading of cellular user sessions onto D2D links in unlicensed bands," IEEE Journal on Selected Areas in Communications, vol. 33, no. 1, pp. 67-80, 2015.

[19] J. E. Wieselthier, G. D. Nguyen, and A. Ephremides, "On the construction of energy-efficient broadcast and multicast trees in wireless networks," in Proceedings of the 19th Annual Joint Conference of the IEEE Computer and Communications Societies (IEEE INFOCOM '00), vol. 2, pp. 585-594, Tel Aviv, Israel, March 2000.

[20] P. Billingsley, Probability and Measure, John Wiley \& Sons, 2012.

[21] M. Crofton, "Probability," in Encyclopedia Britannica, Britannica, 9th edition, 1885.

[22] D. E. Barton, F. N. David, and E. Fix, "Random points in a circle and the analysis of chromosome patterns," Biometrika, vol. 50, no. 1-2, pp. 23-29, 1963.

[23] Y. Wang, Z. Wang, and L. Zhang, "Internet traffic engineering without full mesh overlaying," in Proceedings of the 20th Annual Joint Conference on the IEEE Computer and Communications Societies (IEEE INFOCOM '01), vol. 1, pp. 565-571, Anchorage, Alaska, USA, April 2001. 


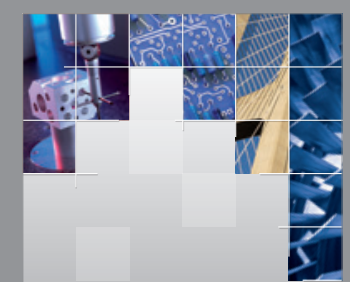

\section{Enfincering}
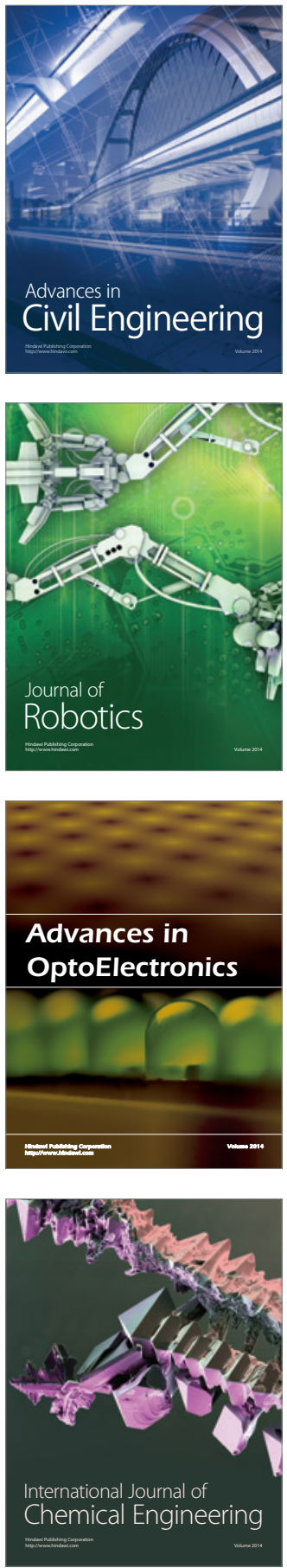

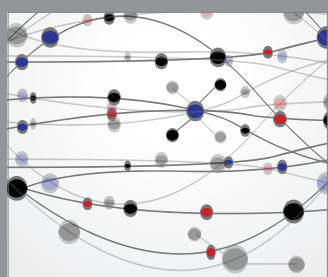

The Scientific World Journal

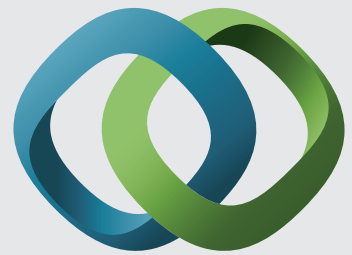

\section{Hindawi}

Submit your manuscripts at

http://www.hindawi.com
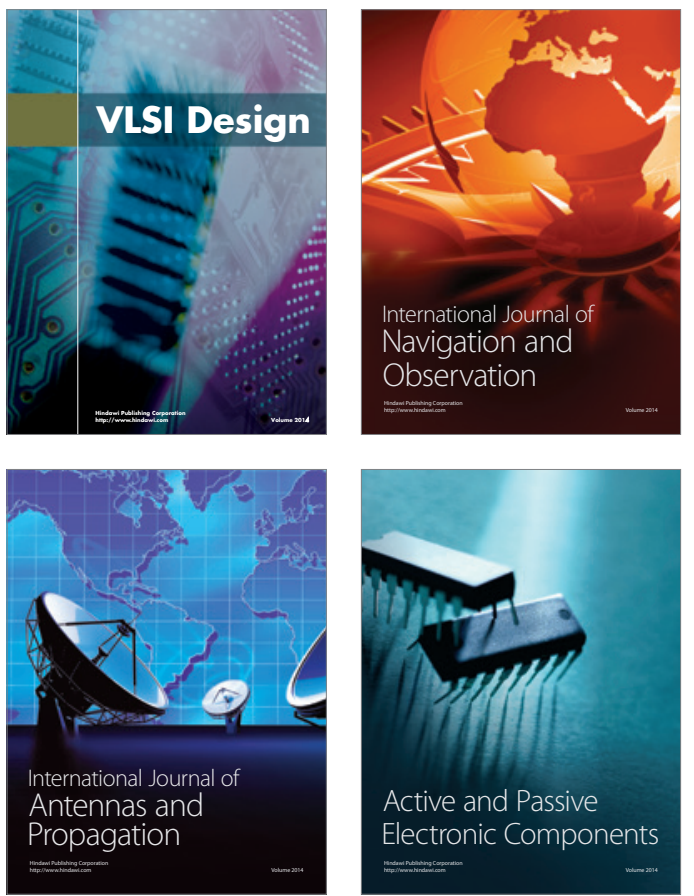
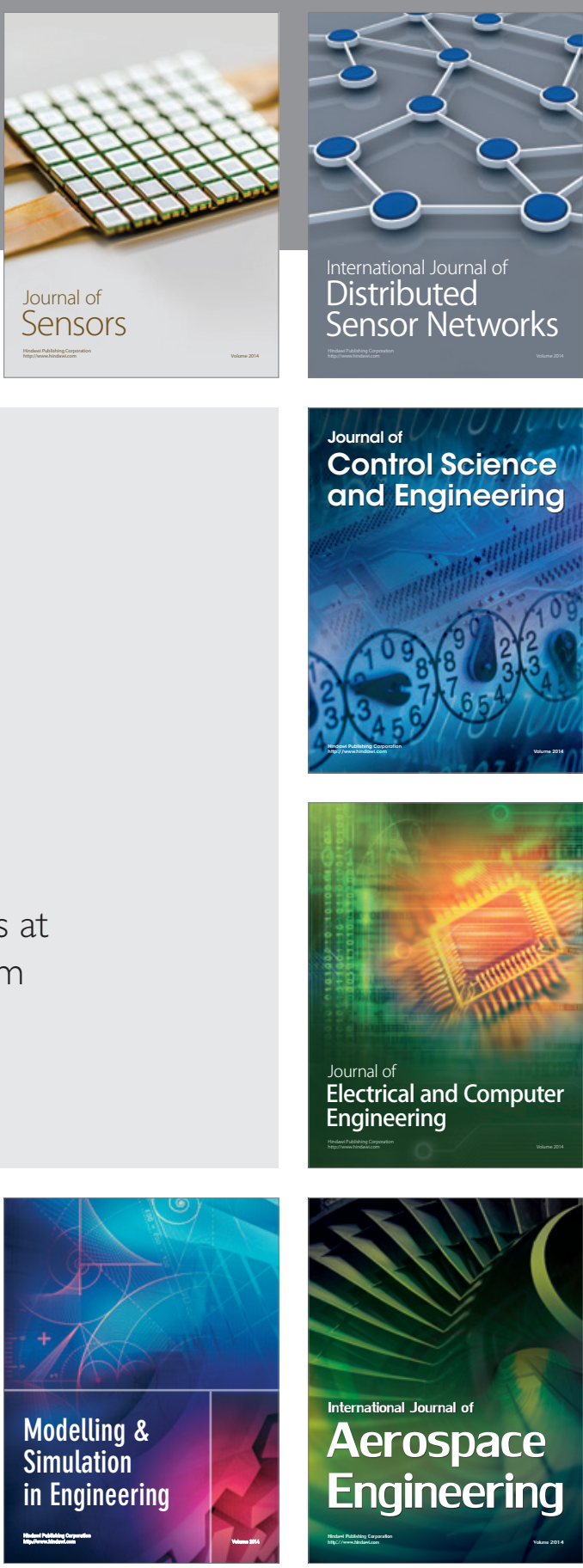

International Journal of

Distributed

Sensor Networks

Journal of

Control Science

and Engineering
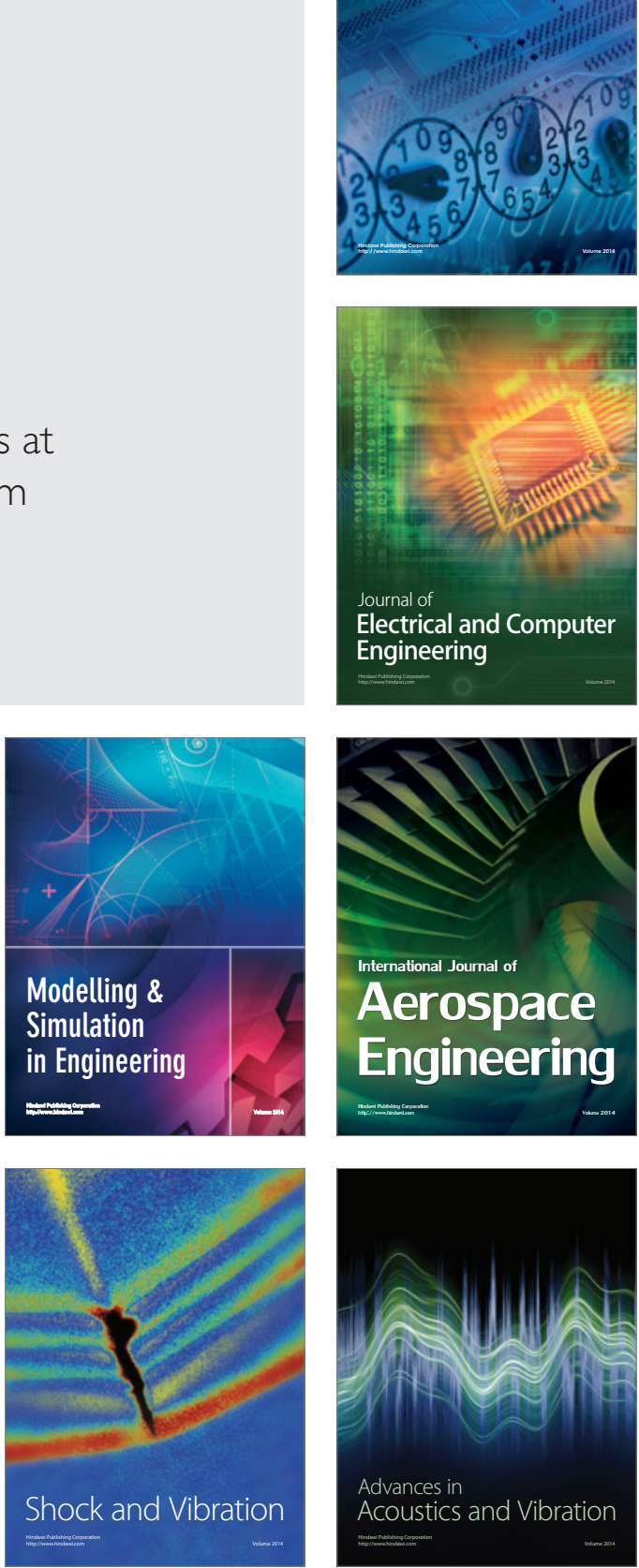